\title{
On the nature of transport in near-critical dissipative-trapped-electron- mode turbulence: Effect of a subdominant diffusive channel
}

\author{
J. A. Mier, ${ }^{1, a)}$ R. Sánchez, ${ }^{2}$ L. García, ${ }^{1}$ D. E. Newman ${ }^{3}$ and B. A. Carreras ${ }^{4}$ \\ ${ }^{1}$ Departamento de Física, Universidad Carlos III de Madrid, 28911 Leganés, Madrid, Spain \\ ${ }^{2}$ Fusion Energy Division, Oak Ridge National Laboratory, Oak Ridge, Tennessee 37831-8071, USA \\ ${ }^{3}$ Department of Physics, University of Alaska, Fairbanks, Alaska 99775-5920, USA \\ ${ }^{4}$ BACV Solutions Inc., Oak Ridge, Tennessee 37830-8222, USA
}

\begin{abstract}
The change in nature of radial transport in numerical simulations of near-critical dissipative-trapped-electron-mode turbulence is characterized as the relative strength of an additional diffusive transport channel (subdominant to turbulence) is increased from zero. In its absence, radial transport exhibits the lack of spatial and temporal scales characteristic of self-organized-critical systems. This dynamical regime survives up to diffusivity values which, for the system investigated here, greatly exceeds the expected neoclassical value. These results, obtained using a novel Lagrangian method, complete and extend previous works based instead on the use of techniques imported from the study of cellular automata [J. A. Mier et al., Phys. Plasmas 13, 102308 (2006)]. They also shed further light on why some features of self-organized criticality seem to be observed in magnetically confined plasmas in spite of the presence of mechanisms which apparently violate the conditions needed for its establishment.
\end{abstract}

\section{INTRODUCTION}

The concept of self-organized criticality (SOC) appeared in the late 1980s as a possible unifying explanation for some of the observed dynamics of very different physical and biological complex systems. ${ }^{1}$ This concept brings together ideas, such as, nonlinear self-organization and critical behavior: critical, because systems in such a state exhibit the same properties that thermodynamical systems in phase transitions, such as, spatial self-similarity, temporal persistence (memory effects) and long-term (divergent) correlations; self-organized, because no external tuning is needed to reach the SOC state. These features are in contrast to those found in purely diffusive systems, characterized by uncorrelated dynamics and spatial and temporal locality.

In the mid 1990s, it was proposed that the SOC paradigm might help to understand some of the observations of nondiffusive, strange radial transport in tokamak plasmas. ${ }^{2,3}$ (These ideas have later been considered for other magnetic confinement devices as well, such as stellarators or reversed-field-pinches. ${ }^{4}$ ) The main requirement for these ideas to be applicable appeared to be the existence of a competition between two mechanisms with clearly separated scales: the external fueling and heating, which push the plasma radial profiles beyond their local instability thresholds; the turbulence which ensues profile flattening and brings them again below threshold. Soon, numerical fluid simulations of near-marginal pressure-gradient-driven plasma turbulence became available which showed that the radial transport indeed exhibited SOC-like features if the aforementioned dynamical cycle is properly captured. ${ }^{5,6}$

\footnotetext{
${ }^{a)}$ Author to whom correspondence should be addressed. Electronic mail: jmier@fis.uc3m.es.
}

However, doubts were also quickly cast upon the relevance of SOC ideas if additional elements, known to be present in magnetically confined plasmas, were also considered. One such example was the presence of an additional diffusive transport channel simultaneously with the near-critical turbulence. ${ }^{7}$ Such a channel is provided by neoclassical diffusion in real plasmas, as well as by any other types of coexisting supercritical turbulence.

In a previous work, ${ }^{8}$ we addressed the relevance of SOC in the presence of subdominant diffusion in the context of numerical simulations of dissipative-trapped-electron-mode (DTEM) turbulence in cylindrical geometry. We found that a considerably strong subdominant diffusivity is required for the system dynamics to cease exhibiting SOC-like features. For the parameters used, this value exceeded the neoclassical value in more than one order of magnitude. The characterization of the transport dynamics in the system was however done by constructing time series of a global (Eulerian) quantity, the turbulent activity, which quantified the turbulent radial transport in the simulation and was then examined with a methodology reminiscent of what is typically used in cellular automata. ${ }^{9}$ This analysis is however not fully satisfactory from a quantitative viewpoint. A more proper characterization of the dynamics would require the study of the (radial part of the) Lagrangian characteristics of the turbulent flow. This study is the objective of the present paper. The influence of diffusion on the dynamics is thus quantified by introducing tracers in the simulations, and characterizing their trajectories for different values of the subdominant diffusivity. The information gathered in this way not only confirms the conclusions previously reported using more qualitative methods, but it also provides new quantitative information from which effective models for radial transport can be constructed. In 


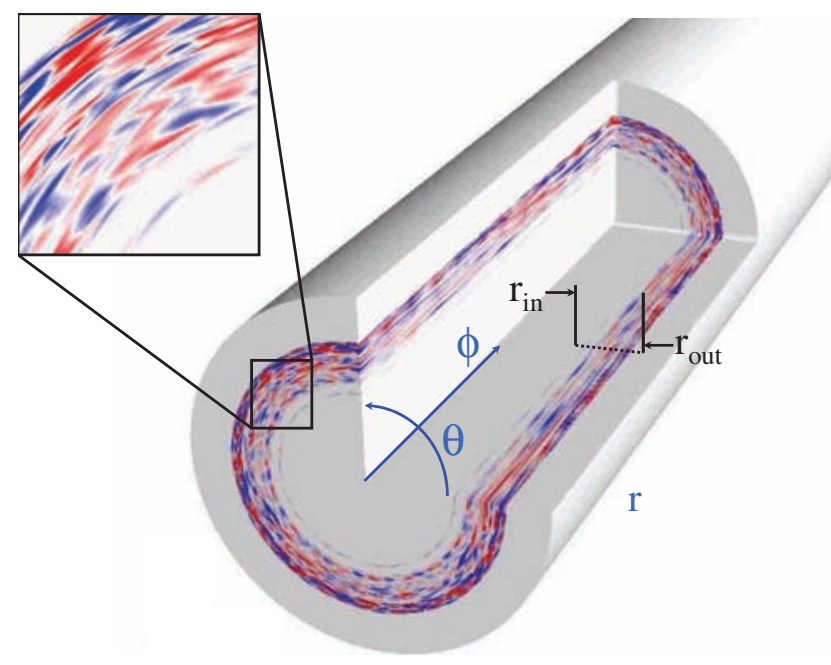

FIG. 1. (Color online) Geometry of the simulations discussed in the text. Inset: snapshot of contours of ion density fluctuations for the $D_{0}=0$ case (red $\rightarrow$ positive; blue $\rightarrow$ negative) .

addition, the method used to characterize the Lagrangian information is novel and quite advantageous with respect to other tracer methods previously used in the literature, most of which are based on random walk analogs.

The paper is organized as follows. In the next section (Sec. II) we review the model used, paying special attention to the elements that ensure the appearance of SOC-like dynamics and to the coupling of the turbulent and the (subdominant) diffusive transport channels. Details about how the tracer particles are advanced will also be provided. Next, in Sec. III, the method that will be used to characterize the tracer trajectories will be presented. The main results obtained from this method will be described in Sec. IV, for different values of the subdominant diffusivity. Finally, in Sec. V some conclusions are drawn.

\section{DISSIPATIVE TRAPPED ELECTRON MODE FLUID MODEL}

The model and numerical implementation used is the same as in Ref. 8. The relevant turbulent mode is the socalled dissipative trapped electron mode (DTEM). ${ }^{10,11}$ The simulations consider a deuterium plasma confined in a periodic cylinder (see Fig. 1) of radius $a=0.5 \mathrm{~m}$ and length $L=2 \pi R_{0}$, with $R_{0}=2 \mathrm{~m}$ so that the equivalent toroid has an inverse aspect ratio $\varepsilon=a / R_{0}=0.25$. To define the position inside the cylinder we use cylindrical coordinates $(r, \theta, Z), r$ being the radius normalized to $a, \theta$ the poloidal angle, and $Z$ the axial position which is related to the toroidal angle $Z=\phi R_{0}$. The plasma is confined by a magnetic field with an axis value of $B=1 \mathrm{~T}$, and a safety factor $q(r)=1.3+0.5 r^{2}$. To derive the equations of the model, ${ }^{10}$ the ions (deuterium) are treated as a cold fluid whilst the electrons are considered under the adiabatic approximation, except for trapped electrons. The latter make the electron density and electrostatic potential fluctuations to be out of phase, a fact which is introduced in the model via an $i \delta$ term (in $k$-space),

$$
\frac{\tilde{n}_{e}}{\bar{n}_{e}}=\frac{e \widetilde{\phi}}{T_{e}}(1-i \delta),
$$

and which ultimately causes the net radial transport. In Eq. (1), $\widetilde{n}_{e}$ and $\bar{n}_{e}$ are, respectively, the fluctuating and average electron densities; $T_{e}$ is the electron temperature; $e$ is the modulus of the electron charge; $\widetilde{\phi}$ is the fluctuating electrostatic potential and $\delta$ is the shift coming from trapped electrons, which can be estimated using gyrokinetic arguments. ${ }^{10}$ Physically, the ad hoc introduction of $\delta$ in Eq. (1) captures in a simple manner the phase difference between density and potential fluctuations caused by the presence of nonadiabatic trapped electrons, which is needed to drive a net outwards radial flux.

\section{A. Equations}

Starting from the ion continuity equation, using Eq. (1) plus quasineutrality, and considering the long-wavelength limit, the model is reduced to two equations describing the time evolution of the fluctuating and the surface-averaged ion densities, $\tilde{n}_{i}(r, \theta, \phi)$ and $\bar{n}_{i}(r)$, respectively. The equation for the first one is ${ }^{10}$

$$
\begin{aligned}
\frac{d \widetilde{n}_{i}}{d t}- & \rho_{s}^{2} \frac{d \nabla_{\perp}^{2} \widetilde{n}_{i}}{d t}+V_{* n} \frac{1}{r} \frac{\partial \widetilde{n}_{i}}{\partial \theta}+D_{\text {eff }} \frac{1}{r^{2}} \frac{\partial^{2} \widetilde{n}_{i}}{\partial \theta^{2}}-\frac{c_{s}^{2}}{\nu_{i}} \nabla_{\|}^{2} \widetilde{n}_{i} \\
& -D_{r} \nabla_{r}^{2} \widetilde{n}_{i}-\mu_{\perp} \nabla_{\perp}^{4} \widetilde{n}_{i}-L_{n} D_{\text {eff }} \\
& \times\left[\nabla_{\perp}\left(\frac{1}{r} \frac{\partial \widetilde{n}_{i}}{\partial \theta}\right) \times \hat{z}\right] \cdot \nabla_{\perp} \widetilde{n}_{i}=0 .
\end{aligned}
$$

Here, $\tilde{n}_{i}$ is normalized to $n_{\mathrm{ax}} \equiv \bar{n}_{i}(0)$. The convective derivative in the first two terms represents the temporal evolution of the fluctuations including the ion polarization drift. The third term is the diamagnetic drift. Next comes the destabilizing term for the drift waves, which is due to the trapped electrons. Indeed, $D_{\text {eff }}$ is antidiffusivity (i.e., negative diffusivity) introduced by the phase shift: $\delta=k_{\theta} D_{\text {eff }} / V_{* n}$, where $V_{* n} \equiv c_{s} \rho_{s} / L_{n}$ is the diamagnetic velocity. Here, $c_{s} \equiv \sqrt{T_{e} / m_{i}}$ is the sound speed, $\rho_{s}=c_{s} / \Omega_{i}$ is the ion Larmor radius, and $L_{n}(r) \equiv \bar{n}_{i}\left|d \bar{n}_{i} / d r\right|^{-1}$ is the density characteristic length. The fifth term provides parallel (to the magnetic field) damping for ions, $\nu_{i}$ being the ion collision frequency. In contrast to all other terms, this term is introduced ad hoc to model parallel collisional damping. The sixth and seventh terms are a numerical diffusivity and hyperviscosity needed to ensure numerical stability. Finally, the last one is a nonlinear term representing the $\mathbf{E} \times \mathbf{B}$ drift convection. Note that, due to the long-wavelength limit taken, no polarization drift nonlinearity is present in this model.

The equation for the surface-averaged density $\bar{n}_{i}$ is ${ }^{8}$

$$
\begin{aligned}
\frac{\partial}{\partial t}\left(\bar{n}_{i}\right. & \left.-\rho_{s}^{2} \nabla_{r}^{2} \bar{n}_{i}\right) \\
= & {\left[S_{0}(r)+\widetilde{S}(r, t)\right]+\nabla_{r}\left[D_{\mathrm{ext}}(r) \nabla_{r} \bar{n}_{i}\right] } \\
& +\left\langle L_{n} D_{\mathrm{eff}}\left[\nabla_{\perp}\left(\frac{1}{r} \frac{\partial \widetilde{n}_{i}}{\partial \theta}\right) \times \hat{z}\right] \cdot \nabla_{\perp} \widetilde{n}_{i}\right\rangle_{\phi, \theta} .
\end{aligned}
$$


The left-hand side of the equation represents the evolution in time of the density profile. The first term of the right-hand side is the source needed to drive the system towards local instability. Otherwise, once the profile flattens and fluctuations are quenched, any turbulent transport would be discontinued. It is composed of two pieces: ${ }^{5}$ a term constant in time, $S_{0}(r)$, chosen so that the steady-state ion density profile in the absence of the nonlinearity is parabolic $\bar{n}_{i}=n_{\mathrm{ax}}\left(1-r^{2}\right)$; the second part of the source, $\widetilde{S}(r, t)$, is random both in time and radius, with zero average and independent of the angular coordinates. The following term on the right-hand side represents the subdominant diffusive channel, which will be treated as a tunable quantity in order to study the dependency of transport dynamics on $D_{\text {ext }}$. [As detailed in Ref. 8, the profile of $D_{\text {ext }}$ is divided in three regions, the middle one being coincident with the interval where turbulence develops (see below). The values on the outer and inner regions are kept fixed and chosen to avoid the piling up of density at the boundaries of that interval. Only the middle diffusivity is varied from simulation to simulation.] The last term, in which the bracket stands for surface-averaging, represents the coupling between the density fluctuations and the mean profile. Note that there is also a coupling in the reverse direction: Any local change in $\bar{n}_{i}$ affects the fluctuations via the density scale length, $L_{n}(r)$, which is hidden in the diamagnetic and nonlinear terms in Eq. (2).

\section{B. Parameters}

Regarding other details specific to the simulations presented here, the axis density value is $n_{\mathrm{ax}}=10^{19} \mathrm{~m}^{-3}$. We consider a plasma with $T_{e}=T_{\mathrm{ax}}\left(1-r^{2}\right)^{2}$, so that the DTEM instability parameter is the same all across the radius $\left(\eta_{e} \equiv L_{n} / L_{T_{e}}=2\right)$. The axis temperature value is $T_{\mathrm{ax}}=2.5 \mathrm{keV}$. The radial region in which rational surfaces (susceptible to become unstable and drive turbulence) are located spans the radial interval $\left[r_{\text {in }}, r_{\text {out }}\right]=[0.48,0.75]$. Within it, 142 Fourier harmonics have been included. This number of angular harmonics may seem low compared with typical simulations of supercritical turbulence in which transport is based uniquely in the energy and enstrophy cascades in $k$-space. In our case, transport is dominated instead by the mean profile relaxation processes, and angular resolution is less important. ${ }^{5}$ We use a radial mesh ${ }^{8}$ with 540 nodes within the interval of interest, $\left[r_{\text {in }}, r_{\text {out }}\right]$, which gives us a radial resolution of $\Delta r=2.5 \times 10^{-4} \mathrm{~m}$. The step size is $\Delta t=50 / \Omega_{i}$, which in physical units is about $1 \mu \mathrm{s}\left(\Omega_{i} \sim 4.8 \times 10^{7} \mathrm{rad} / \mathrm{s}\right)$. Other relevant parameters are listed in Table I. Finally, note that the simulations discussed here have different conditions than those presented in Ref. 8. The safety factor profile is different to allow for a larger radial interval and more optimal distance between rational surfaces. Temperature and field strength are also different. As a result, the width of the computational box is almost three times larger and the ion Larmor radius $\left(\rho_{s}\right)$ is about five times larger. These changes facilitate the simulations with tracers whilst not modifying the qualitative features of the dynamics. However, the result is that the values of $D_{0}$ at which the dynamical changes take
TABLE I. Values of the parameters that characterize the geometry and equilibrium in the numerical calculations at the center of radial interval of interest.

\begin{tabular}{lc}
\hline \hline Parameter & Value \\
\hline Ion gyroradius & $\rho_{s}=4 \times 10^{-3} \mathrm{~m}$ \\
Ion sound velocity & $c_{s}=2.2 \times 10^{5} \mathrm{~m} / \mathrm{s}$ \\
Diamagnetic velocity & $V_{* n}=3.7 \times 10^{3} \mathrm{~m} / \mathrm{s}$ \\
Magnetic shear length & $L_{s}=11 \mathrm{~m}$ \\
Temperature scale length & $L_{T}=0.12 \mathrm{~m}$ \\
Density scale length (equilibrium) & $L_{n}=0.24 \mathrm{~m}$ \\
Ion collision frequency & $\nu_{i}=100 \mathrm{~s}^{-1}$ \\
Trapped electron collision frequency & $\nu_{\text {eff }}=10^{5} \mathrm{~s}^{-1}$ \\
Drive strength & $D_{\text {eff }}=160 \mathrm{~m}^{2} / \mathrm{s}$ \\
Inner diffusivity & $D_{\text {in }}=0.1 \mathrm{~m}^{2} / \mathrm{s}$ \\
Outer diffusivity & $D_{\text {out }}=0.1 \mathrm{~m}^{2} / \mathrm{s}$ \\
\hline \hline
\end{tabular}

place are also different. For that reason, we need to vary $D_{0}$ in the range $0-10^{-7} a^{2} \Omega_{i}$ (approximately $0-1.2 \mathrm{~m}^{2} / \mathrm{s}$ in physical units).

\section{Tracers}

In order to characterize the dynamics of transport at the deepest level, Lagrangian information is required. ${ }^{13,12} \mathrm{Ob}-$ taining such information in a real experiment is however extremely difficult, if not impossible. It can however be routinely obtained in simulations of electrostatic plasma turbulence by following the trajectories of massless test particles (tracers) as they are advected by the time-evolving $E \times B$ electrostatic turbulent flow. ${ }^{14-17}$ The tracer equation of motion is simply

$$
\frac{d \mathbf{r}}{d t}=\mathbf{V}(\mathbf{r}, t)=\mathbf{V}_{\perp}+V_{\|} \frac{\mathbf{B}}{B},
$$

where the perpendicular velocity is given by

$$
\mathbf{V}_{\perp}=\mathbf{V}_{\mathbf{E} \times \mathbf{B}}=-\nabla \tilde{\phi} \times \mathbf{B} / B^{2},
$$

The parallel velocity is in principle arbitrary; in our studies, it is set to zero. Note that the DTEM model prescribed by Eqs. (2) and (3) does not provide the fluctuating electrostatic potential directly. It must be obtained by inverting Eq. (1) as needed.

Regarding particle management, all tracers are started at a single time and only after the simulation has entered into the nonlinearly saturated phase. When discussing time lapses in what follows, they should be understood as measured from this single initial time. All tracers are randomly initialized in space within the intervals, $r_{0} \in[0.50,0.73]$, $\theta_{0} \in[0,2 \pi]$, and $Z_{0} \in\left[0,2 \pi R_{0}\right]$. If a tracer is pushed outside of the radial interval of interest, $\left[r_{\text {in }}, r_{\text {out }}\right]$, it is randomly reallocated within whilst keeping the same identity. As we will see, this prescription helps to squeeze the most information out of the method of analysis used, which we describe in the next section. Note however that this refilling procedure may not be adequate for other analysis methods, for instance, it may be used to analyze flight/waiting-time statistics, ${ }^{14,15,18}$ but not to compute tracer propagators. ${ }^{17}$ This strategy repre- 
sents however a clear advantage, specially in superdiffusive situations in which tracers tend to cross the system boundaries very quickly. Otherwise, an extremely large number of tracers would be required to achieve reasonable statistics.

\section{CHARACTERIZATION OF MOTION: ANALYSIS OF LAGRANGIAN VELOCITIES}

The nature of motion can be characterized using a method based on recent theoretical work ${ }^{12}$ showing that the effective transport equation for the ensemble-average (over flow realization) of any passive scalar advected by a (homogeneous, isotropic, self-similar) turbulent flow can be derived from a proper characterization of the statistical and correlation properties of the flow velocity along its characteristic trajectories.

Namely, suppose a passive scalar (denoted by $n$ ) which is advected by a flow field $\mathbf{V}(\mathbf{r}, t)$ according to the equation

$$
\frac{\partial n}{\partial t}+(\mathbf{V} \cdot \nabla) n=0
$$

and whose transport does not affect the flow in any way. Then, assume $\mathbf{V}=\tilde{\mathbf{V}}$ and $n=n_{0}+\tilde{n}$, where the subindex 0 represents the ensemble-average operation. The flow must have a zero average in order to ensure isotropy. Equation (6) then becomes

$$
\begin{aligned}
& \frac{\partial n_{0}}{\partial t}=-\langle\tilde{\mathbf{V}} \cdot \nabla \tilde{n}\rangle, \\
& \frac{\partial \tilde{n}}{\partial t}+\tilde{\mathbf{V}} \cdot \nabla \tilde{n}=-\tilde{\mathbf{V}} \cdot \nabla n_{0}+\langle\tilde{\mathbf{V}} \cdot \nabla \tilde{n}\rangle,
\end{aligned}
$$

where we also use angular brackets to represent ensembleaveraging. Equation (8) can be solved for the fluctuating part $\tilde{n}$ in terms of $\boldsymbol{\nabla} n_{0}$ and $\tilde{\mathbf{V}}$. Then, inserting the result in Eq. (7), one obtains ${ }^{12}$

$$
\frac{\partial n_{0}}{\partial t}=\nabla \cdot \int_{0}^{t} d t^{\prime}\left\{\left\langle\tilde{\mathbf{V}}(\mathbf{r}, t) \tilde{\mathbf{V}}\left[\mathbf{R}\left(t^{\prime} \mid \mathbf{r}, t\right), t^{\prime}\right] \nabla n_{0}\left[\mathbf{R}\left(t^{\prime} \mid \mathbf{r}, t\right), t^{\prime}\right]\right\rangle\right\} .
$$

The important quantity here is $\tilde{\mathbf{V}}\left[\mathbf{R}\left(t^{\prime} \mid \mathbf{r}, t\right)\right]$, with $\mathbf{R}\left(t^{\prime} \mid \mathbf{r}, t\right)$ representing the location reached by integrating (backwards in time) the differential equation,

$$
\frac{d \mathbf{R}}{d \tau}=\tilde{\mathbf{V}}(\mathbf{R}, \tau), \quad \mathbf{R}(t)=\mathbf{r},
$$

up to time $t^{\prime}<t$. Note that, as $t^{\prime}$ varies, $\mathbf{R}\left(t^{\prime} \mid \mathbf{r}, t\right)$ moves along the characteristic trajectory of the flow. $\tilde{\mathbf{V}}\left[\mathbf{R}\left(t^{\prime} \mid \mathbf{r}, t\right)\right]$ is referred to as the Lagrangian velocity.

The method of analysis we will use later is based on the interesting fact that the ensemble average in Eq. (9) can be estimated, by means of a methodology reminiscent of pathintegral methods, under quite general assumptions regarding the statistical and correlation properties of the Lagrangian velocities along the characteristics of the flow. ${ }^{12}$ These properties thus determine which kind of effective equation will describe (and thus, the nature of) the transport of the ensemble-average of the passive scalar, $n_{0}$. For instance, it is well known that transport is diffusive whenever (1) the Lagrangian velocity becomes uncorrelated of itself quickly (say, after a typical time $\tau_{c}$ ), and (2) it has a finite typical velocity (say, $\left.\widetilde{V}_{c}\right)$. Then, it is straightforward to show that Eq. (9) can be reduced to the familiar diffusive equation,

$$
\frac{\partial n_{0}}{\partial t} \simeq D \frac{\partial^{2} n_{0}}{\partial x^{2}}, \quad D \sim\left(\tilde{V}_{c}^{2} \tau_{c}\right)
$$

for time lapses $\Delta t \gg \tau_{c}$ and distances $|\Delta x| \gg \tilde{V}_{c} \tau_{c}$ (here, we have assumed one dimension for simplicity). The absence of correlations in the Lagrangian velocity endows the transport dynamics with a Markovian character. On the other hand, the Lagrangian velocity must be statistically distributed according to a probability density function (pdf) $P(v)$ with a finite variance $\sigma_{v}^{2}$ in order to have a finite typical value $\left(\tilde{V}_{c} \sim \sqrt{\sigma_{v}^{2}}\right)$. The central limit theorem then predicts that it should follow a Gaussian law, ${ }^{19}$ which makes dynamics inherit a Gaussian character as well. Note that these two properties also introduce a typical transport length $l_{c} \equiv \widetilde{V}_{c} \tau_{c}$, from which $D \sim l_{c}^{2} / \tau_{c} \sim \tilde{V}_{c}^{2} \tau_{c}$, as usual.

In contrast, the transport of $n_{0}$ is no longer well described by the classical diffusive equation whenever the Lagrangian velocities either: ${ }^{20,21}$ (1) exhibit long correlations along the characteristic trajectory, or (2) they lack a finite typical value. One common occurrence is when the long correlations are such that the resulting flow characteristics are self-similar under the transformation $(x, t) \rightarrow\left(\lambda^{H} x, \lambda t\right)$, with a self-similarity exponent $H \in(0,1]$ and with $H \neq 1 / 2$. [In fact, $H=1 / 2$ corresponds to the case with no correlations, since it is the self-similarity exponent of the familiar uncorrelated random walk; ${ }^{19}$ the motion would then reduce to the diffusive one already discussed. For that reason, the case $H<1 / 2$ is usually referred to as anticorrelated and causes subdiffusive transport; similarly, $H>1 / 2$ is referred to as (positively) correlated, and yields superdiffusive transport.] If this hypothesis holds, Eq. (9) reduces to ${ }^{12}$

$$
\frac{\partial n_{0}}{\partial t}={ }_{0} D_{t}^{1-2 H}\left[D^{\prime} \frac{\partial^{2} n_{0}}{\partial x^{2}}\right]
$$

Here, ${ }_{a} D_{t}^{\gamma}$ represents the fractional Riemann-Liouville operator of order $\gamma$ and start point $a . D^{\prime}$ is an effective fractional diffusivity, whose precise definition ${ }^{22}$ will not be needed in what follows. It suffices to say that it is an integral-differential operator that contains integrals over the whole domain of the independent variable (in this case, time). They appear in Eq. (12) because of the long correlations between the Lagrangian velocities, which imply that the motion can never be assumed independent of its past history. In other words, in the diffusive (Gaussian, Markovian) case, past dynamics could be ignored for $t \gg \tau_{c}$; but for $H \neq 1 / 2$, this is not true for any value of $\tau_{c}$ !

If, in addition, a finite typical value for the Lagrangian velocity is also lacking, a finite transport typical length can no longer be defined. Mathematically, this translates into a pdf for the Lagrangian velocities which has an infinite variance. In this case, the central limit theorem predicts ${ }^{19}$ that the 
pdf should be a (symmetric) Lévy law with exponent $\alpha \in(0,2)$. Lévy laws can be expressed in closed analytical form only via their Fourier transform. ${ }^{19}$ In the symmetric case, this expression is $L_{\alpha, \sigma}(k)=\exp \left(-\sigma^{\alpha}|k|^{\alpha}\right)$, where $\sigma$ is a scale factor. However, it follows from their definition that these stable laws decay as $L_{\alpha}(v) \sim|v|^{-(1+\alpha)}$ for large values of their argument, which is the cause of their lack of a finite variance. The transport equation derived from Eq. (9) is, in this case, ${ }^{12}$

$$
\frac{\partial n_{0}}{\partial t}={ }_{0} D_{t}^{1-\alpha H}\left[D^{\prime \prime} \frac{\partial^{\alpha} n_{0}}{\partial|x|^{\alpha}}\right] .
$$

Here, $\partial^{\varphi} n / \partial|x|^{\varphi}$ is the Riesz fractional derivative ${ }^{22}$ of order $\varphi$. It is again an integral-differential operator which contains an integral over the whole spatial domain, and reduces to the usual Laplacian for $\alpha \rightarrow 2$. Its appearance is a reflection of the fact that no location is sufficiently far from a given point to have a negligible effect on transport at that point, since all extreme values of the Lagrangian velocities are statistically meaningful. Note also that the fractional integral in time disappears only if $H=1 / \alpha$, which is the self-similarity exponent for uncorrelated motion if $\alpha<2$.

The method we will use to characterize the nature of transport in our simulations is based on these ideas. Noting that the flow characteristics coincide with the trajectories followed by the tracers, we use them to provide independent realizations (as many as tracers) of Lagrangian velocity time series. Since our interest is on radial transport, we will only examine the correlation and statistical properties of the radial component of the Lagrangian velocities. The shape of their pdf will provide a quantitative estimate for the exponent $\alpha$ (2, if Gaussian; $<2$, otherwise). The self-similarity exponent $H$ will then give a quantitative measure of the degree of correlation $(1 / \alpha$, for uncorrelated; $<1 / \alpha$, if anticorrelated; $>1 / \alpha$, if correlated).

Before proceeding to the next section, it only remains to sketch upon the method used to determine $H$. We will use a variation of the traditional rescaled-range method introduced by Hurst to quantify correlation in Gaussian-distributed series. ${ }^{23}$ The original method consists on the construction of the rescaled-range of the Lagrangian velocity time series, $V_{i}, i=1, \ldots, N$, via

$$
[R / S](\tau) \equiv \frac{\max _{1 \leqslant k \leqslant \tau} W(k, \tau)-\min _{1 \leqslant k \leqslant \tau} W(k, \tau)}{\sqrt{\left\langle V^{2}\right\rangle_{\tau}-\langle V\rangle_{\tau}^{2}}} .
$$

Here, $W(k, \tau) \equiv \sum_{i=1}^{k} V_{i}-k\langle V\rangle_{\tau}$ and $\langle\cdot\rangle_{\tau}$ represents the average up to time $\tau$. When the signal is self-similar, $[R / S] \sim \tau^{H}$ and $H$ is the self-similarity (or Hurst) exponent. The prescription must however be changed for Lévy-distributed entries. Indeed, since their variance is infinite, the denominator of the rescaled-range scales with $\tau$ and distorts the exponent, which would no longer be the self-similarity exponent. The fix is easy: We substitute the square-root of the variance by the $1 /$ sth power of any moment of order $s>0$, with $s<\alpha$, since these are all finite. ${ }^{19}$
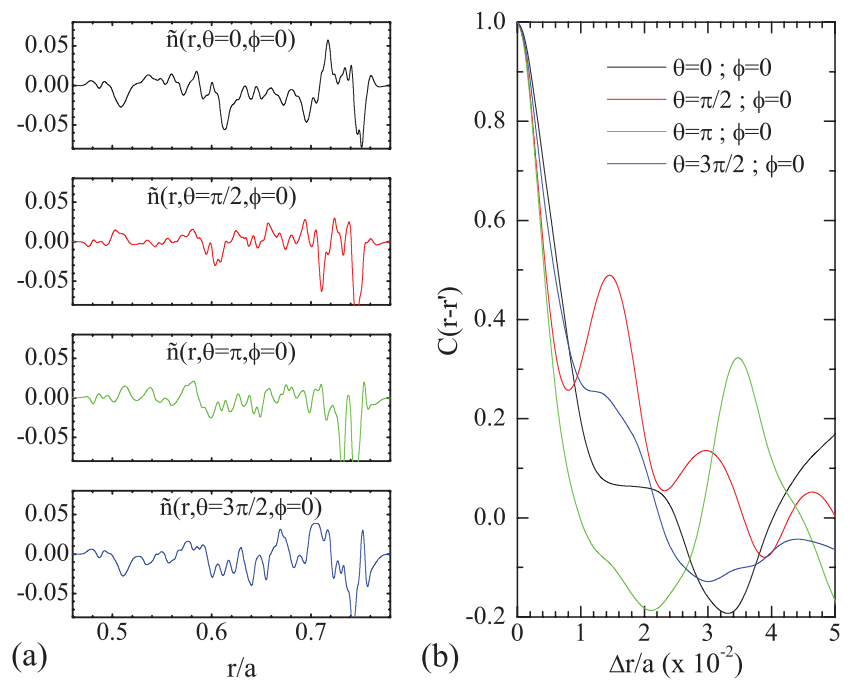

FIG. 2. (Color online) (a) Instantaneous radial fluctuating density profile $\left(D_{0}=0\right.$ case $)$ at $\phi=0$ plane for poloidal positions $\theta=0, \theta=\pi / 2, \theta=\pi$, and $\theta=3 \pi / 2$. (b) Radial autocorrelation functions of the four signals in (a).

\section{ANALYSIS OF THE RESULTS}

We proceed now to describe the results of applying the method described in Sec. III to $\left(\sim 10^{5}\right)$ tracers advected by the DTEM turbulence in four simulations with different values of the subdominant diffusivity, $D_{0}=0,3 \times 10^{-9}$, $1.5 \times 10^{-8}$, and $10^{-7}$, in $a^{2} \Omega_{i}\left(\sim 1.2 \times 10^{7} \mathrm{~m}^{2} / \mathrm{s}\right)$ units. In order to facilitate the interpretation of the results, we first characterize the smallest (radial) length and time scales of the turbulence. This is important because, in real physical systems, self-similarity cannot be expected to hold for arbitrary small and large scales. In our case, it is limited from below by the radial correlation length and the Lagrangian decorrelation time, and from above by the system radius and the shortest of two times: the mean tracer confinement time and the simulation duration. The range of scales over which selfsimilarity may appear must lie between these limiting scales and is usually referred to as the mesoscale. ${ }^{24,25}$ In our simulations, the mesoscale extends for a decade in the spatial domain, whereas in the temporal domain it spans about two decades.

\section{A. Smallest meaningful turbulent scales}

The smallest meaningful spatial scale of the turbulence in the direction of interest (i.e., radial) is given by the radial correlation function of the fluctuating ion density,

$$
C_{\theta_{0}, \phi_{0}}^{\mathrm{rad}}(\Delta)=\frac{1}{\sigma_{\tilde{n}}^{2}} \int d r \tilde{n}_{i}\left(r, \theta_{0}, \phi_{0}\right) \tilde{n}_{i}\left(r+\Delta, \theta_{0}, \phi_{0}\right) .
$$

Here, $\sigma_{\widetilde{n}}^{2}$ is the perturbed ion density variance; $\theta_{0}, \phi_{0}$ are some prescribed poloidal and toroidal angles, and the radial integral is carried out over the interval of interest: $\left[r_{\text {in }}, r_{\text {out }}\right]$. Figure 2(a) shows the radial profiles of the fluctuating ion density and Fig. 2(b) the corresponding correlation functions for different directions in the $\phi_{0}=0$ toroidal plane for the $D_{0}=0$ case. From the decay of these functions, we infer that 
TABLE II. Basic time scales (in units of $\Delta t \sim 1 \mu \mathrm{s}$ )

\begin{tabular}{lcc}
\hline \hline Time scale & $\mathbf{D}_{0}=0$ & $\mathbf{D}_{0}=10^{-7} a^{2} \Omega_{i}$ \\
\hline Lagrangian decorrelation time & 5 & 10 \\
Eulerian decorrelation time & 40 & 60 \\
Confinement time & 130 & 56 \\
Simulation time & $10^{4}$ & $10^{4}$ \\
\hline \hline
\end{tabular}

the radial coherence has a typical value of $\rho_{d} \simeq 0.01 a$, which provides an estimate for the average radial eddy size.

The smallest meaningful temporal scale is given by the Lagrangian decorrelation time, defined as the lapse of time needed for the radial Lagrangian correlation function,

$$
C_{r}^{\mathrm{Lag}}\left(t-t^{\prime}\right) \equiv\left\langle V_{r}\left[\mathbf{R}\left(t \mid \mathbf{r}_{0}, t^{\prime}\right), t\right] V_{r}\left[\mathbf{r}_{0}, t^{\prime}\right]\right\rangle,
$$

to go through zero for the first time. Here, $V_{r}$ represents the radial component of the tracer velocity. Even when $C_{r}^{\mathrm{Lag}}$ appears to depend not only on $t-t^{\prime}$, but also on the initial position $\mathbf{r}_{0}$, that dependence disappears if the turbulence is approximately homogeneous. The Lagrangian decorrelation times vary between $5 \Delta t$ (remember, $\Delta t \simeq 1 \mu \mathrm{s}$ ) for the $D_{0}=0$ case, to $10 \Delta t$ for the largest diffusivity $\left(D_{0}=10^{-7}\right)$.

Other temporal scales of interest are the zero-distance Eulerian decorrelation time, the mean tracer confinement time, and the simulation time. Values for all of them are collected in Table II. The Eulerian time is computed, similarly to what we did in the Lagrangian case, from the zerodistance, radial Eulerian correlation function $C_{r}^{\mathrm{Eul}}\left(t-t^{\prime}\right)$ $\equiv\left\langle V_{r}\left[\mathbf{r}_{0}, t\right] V_{r}\left[\mathbf{r}_{0}, t^{\prime}\right]\right\rangle$, which also becomes independent of $\mathbf{r}_{0}$ after ensemble-averaging.

\section{B. Determination of the fractional exponents $\alpha$ and $H$ via the Lagrangian method}

First, we discuss the exponent $\alpha$. Figure 3(a) shows the Lagrangian velocity pdfs (dotted lines) for all values of the background diffusivity, together with the corresponding Lévy fits (dashed lines). The particle refilling procedure previously discussed has no other effect on the calculation than improving the statistics. The deviation from Gaussianity is strongest for $D_{0}=0$ (upper panel), in which $\alpha \sim 0.98$. A gradual transition from nonlocality $(\alpha<2)$ to Gaussianity $(\alpha=2)$ is apparent as the subdominant diffusivity increases (see also Table III). Note also that the Gaussian character is not fully exhibited even at the largest diffusivity value examined, since $\alpha \sim 1.73$ still provides a better fit to the data than a Gaussian, at least in a chi-square sense.

Regarding the self-similarity exponent, $H$, Fig. 3(b) shows its "instantaneous" value as a function of the time lapse $\tau$ for all values of $D_{0}$. This instantaneous value is computed using $\left(H=\tau[R / S]^{-1} d[R / S] / d \tau\right)$, which is exact for a perfectly self-similar process (since then $[R / S] \propto \tau^{H}$ ). As we mentioned previously, self-similarity should be expected only within the mesoscale range, represented in the plots by vertical lines. The lower limit is (5-9) $\mu$ s, consistent with the Lagrangian decorrelation times. The upper limit is set by the mean confinement time of the tracers, much shorter than the simulation duration (see Table II). No correlation can be
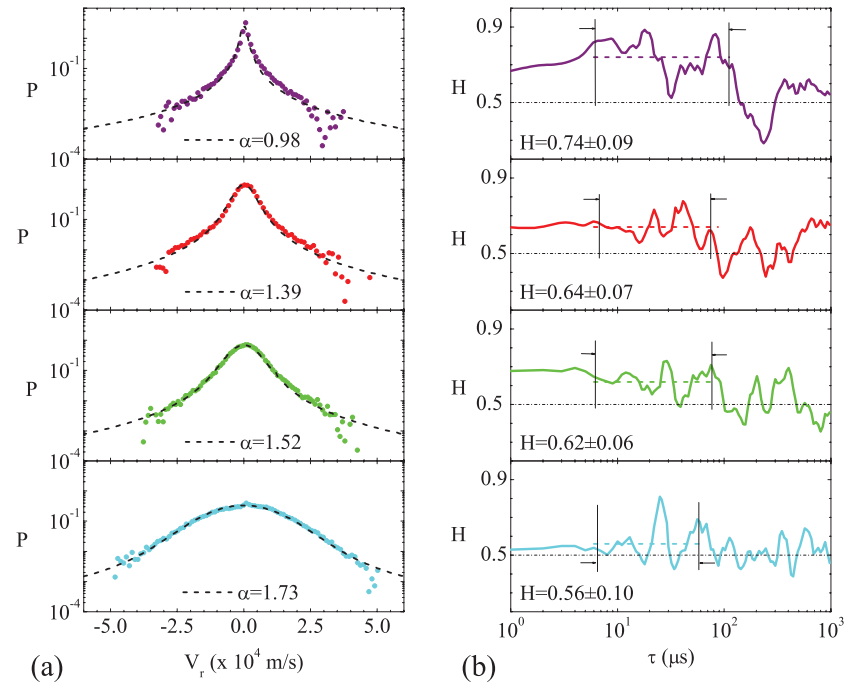

FIG. 3. (Color online) (a) Probability density functions of tracer Lagrangian velocities for different values of the subdominant diffusivity $D_{0}$ (top: $D_{0}=0$; second: $D_{0}=3 \times 10^{-9} a^{2} \Omega_{i}$; third: $D_{0}=1.5 \times 10^{-8} a^{2} \Omega_{i}$; bottom: $D_{0}=10^{-7} a^{2} \Omega_{i}$ ). Best (in a chi-square sense) Lévy fit is shown in dashed black lines for all cases. (b) Hurst exponent of Lagrangian velocity series vs elapsed time for the same cases as in (a). The mesoscale is marked by arrows.

maintained beyond this range since tracers leaving the region are reallocated randomly within. For that reason, the upper end of the mesoscale appears in the plots as a sudden drop of the instantaneous $H$ value towards 0.5 , which is the uncorrelated value, beyond the upper limit. The only case in which this drop is not clearly distinguished from the behavior within the mesoscale is for the largest value of $D_{0}$. As discussed in what follows, the dynamics are already almost diffusive in that case, which means that almost no dynamical difference exists between confined and relocated particle motion. Note also that the fact that particles keep their identity after reallocation works to our advantage here as well, since it improves the statistics of $H$ over the mesoscale range without compromising the validity of the method.

The mean value of $H$ over the mesoscale reveals strong superdiffusive transport $(H \sim 0.74>1 / 2)$ in the case of absence of subdominant diffusion. This result, together with the strongly non-Gaussianity $(\alpha \sim 0.98<2)$, is consistent with the superdiffusive transport via correlated avalanches characteristic of SOC-like dynamics which was previously reported in Ref. 8 for simulations in similar conditions. However, note that the current analysis surpasses the findings of that work, in the sense that the quantitative values for the exponents

TABLE III. Values of $\alpha$, self-similarity exponent $H$ and $\beta(\equiv \alpha H)$ obtained from the Lagrangian method for different values of the background diffusivity $D_{0}$.

\begin{tabular}{lccc}
\hline \hline$D_{0}\left(\times 10^{-7} a^{2} \Omega_{i}\right)$ & $\alpha$ & $H$ & $\beta$ \\
\hline 0 & $0.98 \pm 0.02$ & $0.74 \pm 0.09$ & $0.73 \pm 0.10$ \\
0.03 & $1.39 \pm 0.02$ & $0.64 \pm 0.07$ & $0.89 \pm 0.11$ \\
0.15 & $1.52 \pm 0.02$ & $0.62 \pm 0.06$ & $0.94 \pm 0.10$ \\
1 & $1.73 \pm 0.02$ & $0.56 \pm 0.10$ & $0.97 \pm 0.18$ \\
\hline \hline
\end{tabular}


provide now, by means of Eq. (13), effective radial transport equations. As a curiosity, note also that the Lagrangian velocity series is anticorrelated, since $H \sim 0.74<1 / \alpha(\sim 1.02)$, in spite of yielding superdiffusive transport. This situation, impossible for Gaussian-distributed statistics in which superdiffusion necessarily requires positive correlation, is now possible because of the presence of Lévy statistics.

As the subdominant diffusivity $D_{0}$ increases from zero we find that the self-similarity exponent $H$ decreases. This, combined with the fact that $\alpha$ also tends to the Gaussian value means that transport, in spite of being still mostly carried by turbulence (if an effective diffusivity is computed using $D_{\text {turb }} \sim \widetilde{\Gamma} / \nabla n \sim 10^{-6}$, one order of magnitude larger than the largest $D_{0}$ used), approaches a more diffusivelike behavior, again in consistence with the findings from Ref. 8 . But it is only at the largest $D_{0}$ that such diffusivelike behavior starts to become fully apparent. Indeed, for $D_{0} \sim 10^{-7}$, we find $H \simeq 0.56$ and $\alpha \simeq 1.73$, which implies that $H \sim 1 / \alpha$ $=0.58$, the uncorrelated value. But for smaller diffusivities, SOC-like features are sill dominating the dynamics. To conclude, it is interesting to compare this value of the diffusivity, which in physical units is $D_{0} \sim 1.2 \mathrm{~m}^{2} / \mathrm{s}$, with the neoclassical prediction for the parameters of the simulation, $D_{\text {neo }} \sim \sqrt{2 \epsilon} q^{2} \rho_{s}^{2} \nu_{e i} \sim 5 \times 10^{-3} \mathrm{~m}^{2} / \mathrm{s}$, meaning that neoclassical diffusion is too weak to allow for a significative departure from superdiffusion in our system, at least for the parameter values used in our calculations.

\section{Comparison with CTRW-based methods}

In order to better appreciate the advantages of the Lagrangian method, we proceed now to estimate fractional transport exponents using a more standard method based on the old concept of continuous-time random walks, ${ }^{26,27}$ popularly referred to as CTRWs. The simplest CTRWs model the transport of particles which are assumed to move from their initial position by taking a step (or flight) $\Delta$, with probability $p(\Delta)$, after having waited for a lapse of time $w$, with probability $\psi(w)$. The CTRW is then defined when the two pdfs, $p(\Delta)$ and $\psi(w)$, are prescribed. It is then straightforward to prove that, if $p$ is a Gaussian law with variance $\sigma^{2}$ and $\psi$ an exponential with mean $\mu$, the motion of the density of walkers $n_{w}$ is governed by the classical diffusive equation, ${ }^{20}$

$$
\frac{\partial n_{w}}{\partial t}=B \frac{\partial^{2} n_{w}}{\partial x^{2}}, \quad B \sim \frac{\sigma^{2}}{\mu} .
$$

Note, however, that a finite variance $\sigma^{2}$ implies a finite transport typical length $l \sim \sqrt{\sigma^{2}}$, and that a finite mean waiting-time $\mu$ means that transport becomes essentially Markovian for time lapses $\tau \gg \mu$. If one wants to include non-Gaussian, non-Markovian transport in this context, one needs to choose instead pdfs with infinite variances and/or means. The central limit theorem ${ }^{19}$ suggests again the use of symmetric Lévy pdfs of index $\alpha \in(0,2)$ for $p(\Delta)$. Also within the nonsymmetric members of the Lévy family with index less than 1 , one can find appropriate one-sided pdfs which decay as $\psi(w) \sim w^{-(1+\beta)}$ with $\beta \in(0,1)$ and thus lack a

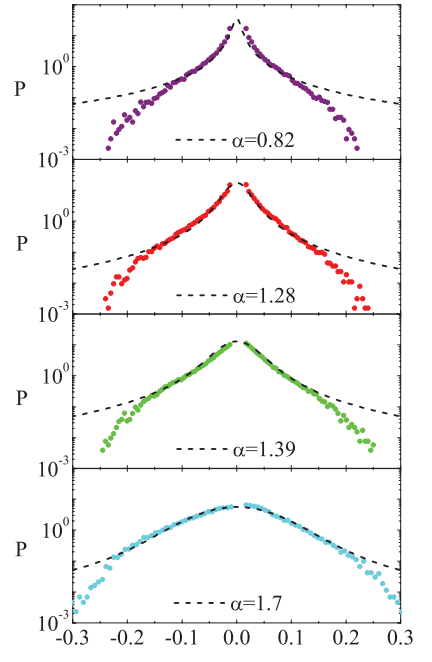

(a)

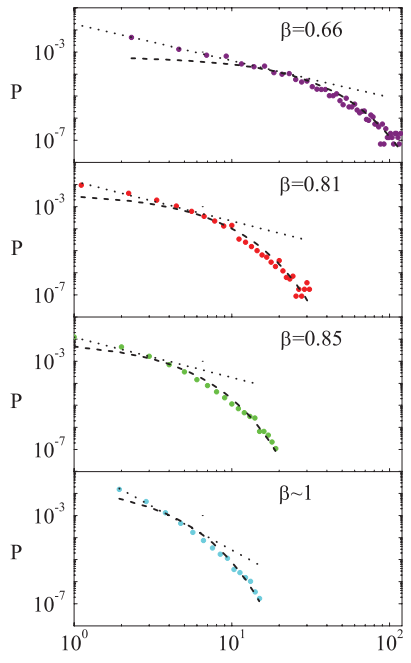

(b)

$\Delta \mathrm{t}$
FIG. 4. (Color online) (a) Probability density functions of flights for different values of the subdominant diffusivity $D_{0}$ (top: $D_{0}=0$; second: $D_{0}=3 \times 10^{-9} a^{2} \Omega_{i}$; third: $D_{0}=1.5 \times 10^{-8} a^{2} \Omega_{i}$; bottom: $D_{0}=10^{-7} a^{2} \Omega_{i}$ ). Best (in a chi-square sense) Lévy fit is shown in dashed black lines for all cases. (b) Probability density function of waiting times for the same values. Best power-law/exponential fit is shown in dotted/dashed black lines for all cases.

finite mean. With these choices, the density of tracers can be lengthily but straightforwardly shown to follow an effective transport equation of the form ${ }^{28,29}$

$$
\frac{\partial n_{w}}{\partial t}={ }_{0} D_{t}^{1-\beta}\left[B^{\prime} \frac{\partial^{\alpha} n_{w}}{\partial|x|^{\alpha}}\right] .
$$

The analysis of the motion of an arbitrary population of particles can then be done after assimilating the motion of the particles of interest (here, the radial motion of tracers) to a CTRW by coming up with a suitable definition of what constitute a flight and a waiting-time. Then, one constructs their pdfs and determines the two tail exponents $\alpha$ and $\beta$. If $\alpha<2$ and/or $\beta<1$, transport is nondiffusive. Note, however, that there are important (if subtle) differences between this method and the Lagrangian one, in spite of the fact that Eqs. (13) and (18) are formally identical after equating $\beta=\alpha H$. First, note that $n_{w}$ in Eq. (18) represents the actual density of the tracers being used to expose the flow characteristics, whilst $n_{0}$ in Eq. (13) represents the ensemble-average of the advected passive scalar. Secondly, $\beta \leqslant 1$ in Eq. (18) due to some restrictions imposed by the CTRW construction, ${ }^{12,20}$ whilst $\alpha H \leqslant 2$ in Eq. (13). The equations for $1<\alpha H<2$ are related to the so-called fractional wave equation, ${ }^{30}$ which are essentially different from a CTRW.

Ignoring these subtleties, we proceed to apply the CTRW method to the tracer data. An additional difficulty appears at this point since, in the case of turbulent simulations, tracers are never at rest. Some definition of what constitutes a jump and a waiting time must be provided by the user, and its appropriateness may affect the outcome of the analysis. Here, we will use those proposed in Ref. 30, although others have also been used in the literature. ${ }^{14,15,31}$ They are based on the intuitive idea that tracers are "trapped" while circulating around some eddy, and execute a jump 
TABLE IV. Values of $\alpha, \beta$ and the self-similarity exponent $H \equiv \beta / \alpha$ obtained using the CTRW method for different values of the background diffusivity $D_{0}$

\begin{tabular}{lccc}
\hline \hline$D_{0}\left(\times 10^{-7} a^{2} \Omega_{i}\right)$ & $\alpha$ & $H$ & $\beta$ \\
\hline 0 & $0.82 \pm 0.07$ & $0.80 \pm 0.17$ & $0.66 \pm 0.09$ \\
0.03 & $1.28 \pm 0.06$ & $0.63 \pm 0.11$ & $0.81 \pm 0.10$ \\
0.15 & $1.39 \pm 0.07$ & $0.61 \pm 0.12$ & $0.85 \pm 0.12$ \\
1 & $1.70 \pm 0.06$ & $\sim 0.5$ & $\sim 1$ \\
\hline \hline
\end{tabular}

when they move radially over more than one eddy. In terms of the radial component of their Lagrangian velocity, it should then be expected that the radial velocity would stay small (and varying sign rapidly) while trapped, and become large (with a well defined sign) while jumping. Then, it is sufficient to choose an appropriate threshold value, and define the flight as the radial distance moved while the velocity stays over that value; similarly, a waiting time is defined as the time that the velocity stays below the threshold. ${ }^{32}$ (For completeness, we have also tried another usual definition for flights: The radial distance moved while velocity maintains its $\operatorname{sign}^{31}$ which, at least in our case, yields a very similar value for the $\alpha$-exponent.)

Results are shown in Fig. 4. Frame (a) shows the pdfs of the flights for all diffusivities, together with the best symmetric Lévy fits in dashed lines. Frame (b) shows the pdfs of the waiting times, together with best power-law fits. In contrast to the results from the Lagrangian method, a large distortion is apparent in the tails of all pdfs. This is due in part to subsampling problems associated with the system finite-size, and in part to the definitions used. For instance, in the case of the flights, particles leaving the computational box from positions near to the center cannot perform flights bigger than half the total radial size of the computational box $(0.27 a)$, but these flights can be performed by particles closer to the boundaries (at least, with one sign). This situation translates into a subsampling of the tails, and thus all Lévy fits have been done considering only values up to $\pm 0.15 a$. The waiting-times also exhibit strong distortions, that appear as an exponential tail at large values. Although the central part can still be used to estimate $\beta$ (except for the largest diffusivity), the method is clearly far from satisfactory.

The values of $\alpha$ and $\beta$ obtained with the CTRW method have been collected in Table IV, together with the value of the self-similarity exponent computed using $H=\beta / \alpha$. In spite of the difficulties mentioned (and the subtle differences of interpretation discussed previously), the results are consistent with the values of $\alpha$ and $H$ (and the derived exponent $\beta=\alpha H$ ) obtained in the previous section with the Lagrangian method (see Table III). But we feel that the advantages and cleanliness of the Lagrangian method in the context of turbulence, especially in superdiffusive cases in which tracers stay confined for short times, are apparent. It should be noted that this is not a limitation of the CTRW methods themselves and that, in cases in which the limited system size is not so important (i.e., subdiffusive transport), they should perform similarly well.

\section{CONCLUSIONS}

In this paper we have analyzed numerical simulations of DTEM plasma turbulence in cylindrical geometry, in the presence of an additional subdominant diffusive channel, in order to study the persistence of SOC-like features in the dynamics as a function of the subdominant diffusion. The variations in the nature of radial transport have been determined by characterizing the changes in the statistical and correlation properties of the radial components of the flow velocity along the characteristics of the flow for various subdominant diffusivity values. We have shown that this method provides a robust way to estimate fractional transport exponents in the context of numerical plasma turbulence, with multiple advantages with respect to other methods already existent in the literature.

\section{ACKNOWLEDGMENTS}

The authors express their thanks to José Ramón MartínSolís (Universidad Carlos III de Madrid, SPAIN) and Boudewijn Ph. van Milligen, Iván Calvo, and Guillermo Sánchez-Burillo (CIEMAT, Madrid, SPAIN) for very stimulating discussions.

This research was sponsored by DGICYT (Dirección General de Investigaciones Científicas y Tecnológicas) of Spain under Project No. ENE2006-15244-C03-01/FTN. Research sponsored in part by the Laboratory Research and Development Program of Oak Ridge National Laboratory, managed by UT-Battelle, LLC, for the U.S. Department of Energy under Contract No. DE-AC05-00OR22725. Research supported in part by DOE Office of Science Grant No. DEFG02-04ER5741 at the University of Alaska.

${ }^{1}$ P. Bak, C. Tang, and K. Weisenfeld, Phys. Rev. Lett. 59, 381 (1987)

${ }^{2}$ P. H. Diamond and T. S. Hahm, Phys. Plasmas 2, 3640 (1995).

${ }^{3}$ D. E. Newman, B. A. Carreras, P. H. Diamond, and T. S. Hahm, Phys. Plasmas 3, 1858 (1996).

${ }^{4}$ E. Spada, V. Carbone, R. Cavazzana, L. Fattorini, G. Regnoli, N. Vianello, V. Antoni, E. Martines, G. Serianni, M. Spolaore, and L. Tramontin, Phys. Rev. Lett. 86, 3032 (2001).

${ }^{5}$ B. A. Carreras, D. E. Newman, V. E. Lynch, and P. H. Diamond, Phys. Plasmas 3, 2903 (1996)

${ }^{6}$ Y. Sarazin and Ph. Ghendrih, Phys. Plasmas 5, 4214 (1998).

${ }^{7}$ X. Garbet and R. E. Waltz, Phys. Plasmas 5, 2836 (1998).

${ }^{8}$ J. A. Mier, R. Sánchez, L. García, and D. E. Newman, Phys. Plasmas 13 102308 (2006).

${ }^{9}$ R. Sanchez, D. E. Newman, and B. A. Carreras, Nucl. Fusion 41, 247 (2001).

${ }^{10}$ B. A. Carreras, K. Sidikman, P. H. Diamond, P. W. Terry, and L. Garcia, Phys. Fluids B 4, 3115 (1992).

${ }^{11}$ Y. M. Liang, P. H. Diamond, X. H. Wang, D. E. Newman, and P. W. Terry, Phys. Fluids B 5, 1128 (1993).

${ }^{12}$ R. Sanchez, B. A. Carreras, D. E. Newman, V. E. Lynch, and B. Ph. van Milligen, Phys. Rev. E 74, 016305 (2006).

${ }^{13}$ W. D. McComb, Theory of Fluid Turbulence (Oxford University Press, Oxford, 1990).

${ }^{14}$ B. A. Carreras, V. E. Lynch, and G. M. Zaslavsky, Phys. Plasmas 8, 5096 (2001).

${ }^{15}$ L. Garcia and B. A. Carreras, Phys. Plasmas 13, 022310 (2006).

${ }^{16}$ T. Hauff and F. Jenko, Phys. Plasmas 13, 102309 (2006).

${ }^{17}$ D. del-Castillo-Negrete, B. A. Carreras, and V. E. Lynch, Phys. Plasmas 11, 3854 (2004).

${ }^{18}$ G. Spizzo, R. B. White, and S. Cappello, Phys. Plasmas 14, 102310 (2007).

${ }^{19}$ G. Samorodnitsky and M. S. Taqqu, Stable Non-Gaussian Processes (Chapman \& Hall, New York, 1994) 
${ }^{20}$ R. Metzler and J. Klafter, Phys. Rep. 339, 1 (2000).

${ }^{21}$ G. M. Zaslavsky, Phys. Rep. 371, 461 (2002).

${ }^{22}$ I. Podlubny, Fractional Differential Equations (Academic, New York, 1998).

${ }^{23}$ H. E. Hurst, Trans. Am. Soc. Civ. Eng. 116, 770 (1951).

${ }^{24}$ B. A. Carreras, B. Ph. van Milligen, C. Hidalgo, R. Balbin, E. Sanchez, I. Garcia-Cortes, M. A. Pedrosa, J. Bleuel, and M. Endler, Phys. Rev. Lett. 83, 3653 (1999).

${ }^{25}$ R. Sanchez, B. Ph. van Milligen, D. E. Newman, and B. A. Carreras, Phys. Rev. Lett. 90, 185005 (2003).

${ }^{26}$ E. W. Montroll and G. H. Weiss, J. Math. Phys. 6, 167 (1965).
${ }^{27}$ B. Ph. van Milligen, R. Sanchez, and B. A. Carreras, Phys. Plasmas 11, 2272 (2004).

${ }^{28}$ A. Compte, Phys. Rev. E 53, 4191 (1996)

${ }^{29}$ R. Sanchez, B. Ph. van Milligen, and B. A. Carreras, Phys. Rev. E 71, 011111 (2005).

${ }^{30}$ R. Metzler and J. Klafter, Europhys. Lett. 51, 492 (2000).

${ }^{31}$ A. B. Rechester and M. N. Rosenbluth, Phys. Rev. Lett. 40, 38 (1978).

${ }^{32}$ G. Sanchez-Burillo, B. Ph. van Milligen, and A. Thyagaraja, in Proceedings of the 35th EPS Conference on Plasma Physics, Hersonissos, 9-13 June 2008, ECA Vol. 32, p. 2.039 (2008). 